\title{
Should methotrexate have any place in the treatment of psoriatic arthritis?
}

Weiyu Ye ${ }^{1}$, BA MB BChir, Laura C Coates², MB ChB MRCP PhD

\section{Affiliations:}

1. Oxford University Clinical Academic Graduate School, University of Oxford, Room 3A31, The Cairns Library IT Corridor, Level 3, John Radcliffe Hospital (Main Hospital), Oxford, OX3 9DU, UK. christina.ye@medsci.ox.ac.uk

2. Nuffield Department of Orthopaedics, Rheumatology and Musculoskeletal Sciences, Botnar Research Centre, Windmill Road, Oxford, OX3 7LD, UK. laura.coates@ndorms.ox.ac.uk

\section{Corresponding author:}

Dr Laura C Coates

laura.coates@ndorms.ox.ac.uk

Key words:

Methotrexate, psoriatic arthritis, psoriasis, treatment, efficacy

\section{Key points:}

- Methotrexate (MTX) is commonly used in the management of psoriatic arthritis

- There is a paucity of high-quality evidence supporting the efficacy of MTX in psoriatic arthritis, as the available data mainly stems from historical trials with small sample sizes and limited study designs

- $\quad$ MTX may improve joint symptoms in peripheral arthritis, however may not halt radiographic progression

- $\quad$ MTX is effective in treating psoriasis, and may have a beneficial effect in enthesitis, dactylitis and nail disease

- There is limited data on how MTX exerts its beneficial effect in PsA, however this is likely to be through immunomodulation 


\section{Synopsis:}

Methotrexate (MTX) is widely used in the treatment of psoriatic arthritis (PsA) despite the evidence base for this being limited. This narrative review summarises the evidence to date of using MTX within different domains of psoriatic disease, including peripheral arthritis, axial disease, dactylitis, enthesitis, psoriasis and nail disease. We also explore the role of MTX in combination therapy with tumour necrosis factor inhibitors, in addition to its safety and tolerability, to answer the question: should methotrexate have any place in the treatment of psoriatic arthritis?

\section{Disclosure statement:}

Funding: Weiyu Ye is an academic foundation trainee. Laura C Coates is funded by a National Institute for Health Research Clinician Scientist award. The research was supported by the National Institute for Health Research (NIHR) Oxford Biomedical Research Centre (BRC). The views expressed are those of the authors and not necessarily those of the NHS, the NIHR or the Department of Health.

Conflict of interest: Weiyu Ye has no conflict of interest to declare. Laura C Coates has received research funding from Abbvie, Celgene, Lilly, Novartis and Pfizer. Laura C Coates has received honoraria from Abbvie, Amgen, Celgene, Galapagos, Gilead, Janssen, Lilly, Novartis, Pfizer, Prothena, Sun Pharma, and UCB.

\section{Word count:}




\section{Introduction}

Psoriatic arthritis (PsA) is a chronic inflammatory arthropathy with a significant impact on patients’ quality of life ${ }^{1}$. Within PsA, there are several different 'domains' of disease, including peripheral arthritis, axial disease, dactylitis, enthesitis and skin/nail disease, which may vary between different individuals.

Current management is increasingly shifting towards a 'treat-to-target' approach, aimed at controlling disease activity through early intervention and regular objective assessments of disease activity, with escalation in therapy if disease control is not achieved. This strategy stems from the results of the TICOPA study ${ }^{2}$, an openlabel multicentre randomised controlled trial of 206 patients with early PsA. The authors demonstrated that patients in the tight control group were more likely to achieve an American College of Rheumatology 20\% improvement criteria (ACR20) at 48 weeks compared to patients in the standard care group. In practice, this requires a full assessment of the different disease domains to enable appropriate therapy effective against the relevant domains to be initiated. In addition, it also requires assessment of overall response, for example through the minimal disease activity (MDA) criteria ${ }^{3}$.

Currently, methotrexate (MTX) is widely used as the initial therapy in the treatment of PsA, despite limited evidence from high-quality randomised-controlled trials to support this approach ${ }^{4,5}$. This is likely due to the assumed effectiveness of MTX from studies in rheumatoid arthritis (RA) and psoriasis. It is highly unlikely that any further placebo-controlled trials assessing the effectiveness of MTX in PsA will be conducted in the future. This is because it defies clinical equipoise and would be extremely hard to recruit for and it is ethically difficult to justify giving placebo to patients with active PsA when multiple effective treatments for PsA exist.

In this narrative review, we summarise the evidence to date of using MTX in PsA for individual disease domains, as well as in combination with biologic therapy. We also explore the tolerability and adverse effects associated with MTX use in PsA, aiming to answer the question: should MTX be used in the treatment of PsA?

\section{Efficacy of MTX in different domains of PsA}




\subsection{Peripheral arthritis}

MTX is widely used as a conventional synthetic disease modifying anti-rheumatic drug (csDMARD) to treat peripheral arthritis in PsA. However, there is conflicting evidence on whether it is efficacious.

The first double-blind randomised controlled trial of MTX versus placebo was published by Black et al. in 1964 ${ }^{6}$, using a cross-over design. High doses of parenteral MTX were used (1-3 mg/kg) in 21 patients with active peripheral PsA. Compared to placebo, MTX resulted in significant improvements in the joint index (comprising of joint motion, tenderness and swelling), joint range of motion, and erythrocyte sedimentation rate (ESR). There was also a significant reduction in the percentage of skin area affected. However, there was a high incidence of adverse events (AEs), with one patient death. This study was the first to demonstrate that high dose parenteral MTX could improve clinical joint symptoms in PsA, albeit with a substantial risk of AEs.

Several subsequent observational open-label studies have supported the findings of Black et al., demonstrating that MTX can lead to a clinical improvement in joint symptoms in PsA. In a single-centre longitudinal observational study of 59 patients with PsA ${ }^{7}, 68 \%$ exhibited a $40 \%$ or greater reduction in joint count. In this study, patients were treated with a mean MTX dose of $16.2 \mathrm{mg} /$ week, for at least 24 months. Similarly, in an open-label randomised controlled trial of 35 patients with early PsA ${ }^{8}$, patients treated with MTX and nonsteroidal anti-inflammatory drug (NSAID) combination therapy had a greater improvement in their swollen and tender joint count compared to the NSAID monotherapy group at 3 months. These findings are echoed in Lie et al. $2010^{9}$, who published data on 430 patients with PsA treated with MTX monotherapy, collected as part of the NOR-DMARD registry. The mean MTX starting dose was $10.5 \mathrm{mg}$, increasing to $13.7 \mathrm{mg}$ at 6 months. At 6 months, patients with PsA improved in most disease activity measures and patient reported outcomes. Comparing this to patients with RA treated with MTX in the same registry ( $\mathrm{n}=1218)$, patients with PsA tended to have less improvement, although changes were in the same range. Moreover, $65 \%$ of patients with PsA were maintained on MTX by 2 years, compared to $66 \%$ of patients with RA. However, it is important to note that the outcome measures used in this study were more relevant to RA than PsA, for example, the DAS28. 
Furthermore, there is also some evidence that MTX can lead to improvements in composite scores.

Baranauskaite et al. 2012 undertook the RESPOND study ${ }^{10}$, an open-label randomised controlled trial of 115 MTX-naïve patients, comparing MTX monotherapy to MTX plus infliximab combination therapy. MTX was administered at a dose of $15 \mathrm{mg} /$ week. Although combination therapy with infliximab was found to be superior at 16 weeks, the ACR responses for the monotherapy group were relatively high: ACR20 66.7\%, ACR50 39.6\% and ACR70 18.8\%. Furthermore, 24.1\% of patients also achieved MDA with MTX monotherapy. These findings are echoed in a post-hoc analysis of MTX efficacy in the TICOPA study by Coates and Helliwell 2016 11. The TICOPA study was an open-label multicentre randomised controlled trial comparing tight control of PsA versus standard care. Patients in the study had $<24$ months of symptom duration, and were DMARD naïve. In the tight control arm, MTX monotherapy was the first DMARD used for the first 12 weeks unless contraindicated. MTX doses started at $15 \mathrm{mg} /$ week for 4 weeks then escalated to $25 \mathrm{mg} /$ week. In the standard care arm, MTX was used at the discretion of the treating physician. In the first 12 weeks of the trial, 188 patients received MTX, with 104 receiving a mean dose of >15 mg/week. The ACR outcomes at 12 weeks were: ACR20 40.8\%, ACR50 18.8\% and ACR70 8.6\%. Notably, there was a significant reduction in individual tender and swollen joints. Using the psoriatic arthritis disease activity score (PASDAS) response criteria, 57.4\% achieved a moderate/good response, and 18.5\% achieved a good response. Moreover, 22.4\% of patients achieved MDA. Interestingly, there was a trend towards higher proportions of patients taking $>15 \mathrm{mg} /$ week of MTX achieving ACR20, ACR50 and a PASDAS moderate response. However, this is difficult to assess, since patients with well-controlled disease may be maintained on lower doses. Both RESPOND and TICOPA showed that MTX can improve joint outcomes in PsA, with approximately a fifth of patients achieving MDA. However, both studies are limited by their open-label nature and lack of a placebo comparator. Indeed, a small double-blind placebo-controlled randomised controlled trial by Willkens et al. $1984{ }^{12}$ found that MTX was superior to placebo in improving skin psoriasis and physician assessment of disease activity, however was not significantly better at improving joint swelling or tenderness, morning stiffness or patient assessment of disease activity. This study recruited 37 patients with active peripheral PsA $\geq 6$ months duration. MTX was given orally, at a dose of 7.5-15 mg/week, spread over three doses.

In a recent phase 3 randomised multicentre double-blind study (SEAM-PsA) by Mease et al. $2018^{13}, 851$ patients with active PsA and who were naïve to MTX and biologics were randomised into 3 groups: MTX monotherapy plus injectable placebo ( $n=284)$, etanercept monotherapy plus oral placebo ( $n=284)$, and MTX 
plus etanercept combination therapy ( $\mathrm{n}=283$ ). In this cohort, the median PsA duration was 0.6 years (mean of 3.2 years). MTX was administered orally, at a dose of $20 \mathrm{mg} /$ weekly, and from week 4 to 24, the MTX containing arms maintained a mean MTX dose >18.8 mg. At 24 weeks, ACR responses for the MTX monotherapy group were: ACR20 50.7\%, ACR50 30.6\%, and ACR70 13.8\%. 22.9\% of the MTX monotherapy group achieved MDA, with a mean decrease in PASDAS of 2 from baseline. Attainment of MDA has clinical significance, as it correlates with patients reporting low impact of disease, in addition to better functional and radiographic outcomes ${ }^{14-17}$.

In addition to evidence that MTX can lead to improved ACR outcomes and achievement of MDA, a study by Cantini et al. $2008{ }^{18}$ also suggests that MTX can lead to remission in PsA. The authors analysed 121 patients with PsA on MTX monotherapy, at a dose of 10-15 mg/week. They used a very stringent definition of remission, which required the following criteria to be met: fatigue (VAS 1-100mm) < 10, pain (VAS 1-100mm) $<10$, articular morning stiffness $\leq 15$ minutes, tender joint count $=0$, swollen joint count $=0$, ESR $\leq 30$ for women and 20 for men, C-reactive protein $(\mathrm{CRP}) \leq 0.5$, absent dactylitis, enthesitis/tenosynovitis, inflammatory spinal pain, and extra-articular features. There were 23 episodes of remission in patients on MTX monotherapy, demonstrating that remission with MTX is possible in a small number of patients. The ACR responses were AR20 41\%, ACR50 28\% and ACR70 12\%, respectively.

Despite the clinical improvement in symptoms seen with MTX, evidence from randomised-controlled trials and observational studies suggests that MTX has limited disease modifying effect in PsA. Scarpa et al. $2008^{8}$ conducted an open-label randomised controlled trial of 35 patients with early PsA, comparing NSAID monotherapy to NSAID plus MTX combination therapy. Although the MTX group had greater improvement in joint symptoms at 3 months, there was no difference between the two groups in terms of inflammatory markers, or patient and physician assessments of disease activity. Chandran et al. $2008{ }^{7}$ published a single-centre retrospective observational study of 59 patients with PsA treated with MTX for $\geq 24$ months over a 10-year period. They found that although two thirds of patients demonstrated an improvement in skin and joint symptoms, there was nevertheless radiographic progression with a mean increase of 1.5 in the radiographic score using the modified Steinbrocker method. Interestingly, compared to a previous study in the same clinic ${ }^{19}$, Chandran et al. 2008 demonstrated that a greater proportion of patients achieved $\geq 40 \%$ reduction in joint counts 
(68\% vs 47\%), with reduced radiographic progression (1.5 vs 2.3) at 24 months. This was associated with MTX use earlier (mean disease duration 8 years vs 11.5 years), and at higher doses of MTX (16.2 mg/week vs 10.8 mg/week) which may account for better outcomes. However, a cohort analysis undertaken by Eder et al. $2014{ }^{20}$ demonstrated again that MTX therapy does not halt radiographic progression in PsA, even if it does partially affect natural progression. Of 50 patients, 34 (68\%) developed a new erosion in at least one joint and 40 (80\%) had radiographic progression at 1-2 years using the modified Steinbrocker score. By 3-4 years, 42 (84\%) patients had developed a new erosion in at least one joint, and 44 (88\%) had radiographic progression. Similarly, SEAM-PsA ${ }^{13}$ used the van der Heijde modified Total Sharp Score (mtSS), a different radiographic scoring system, to show that $10.6 \%$ of patients treated with MTX monotherapy had radiographic progression from baseline by week 48, with a mean change of 0.08 .

Given the controversy surrounding the efficacy of MTX in PsA, the Methotrexate in Psoriatic Arthritis (MIPA)

${ }^{21}$ trial was conducted, starting in 2003. Published in 2012, it is the largest randomised placebo-controlled trial of MTX in PsA to date. The authors compared MTX at a dose of $15 \mathrm{mg} /$ week to placebo in 221 patients with active peripheral PsA. The dose of MTX was increased slowly, from $7.5 \mathrm{mg} /$ week, to $10 \mathrm{mg} /$ week at 4 weeks and $15 \mathrm{mg} /$ week at 8 weeks. There was an option to increase the MTX dose to $20 \mathrm{mg} /$ week at 4 months, and 25 $\mathrm{mg} /$ week at 5 months, at the discretion of the supervising rheumatologist. The primary outcome was the PsA response criteria (PsARC) at 6 months, which showed no significant effect with MTX compared to placebo (OR 1.77, 95\% CI 0.97, 3.23). Moreover, there was no significant effect of MTX compared to placebo on ACR20 (OR 2.00, 95\% CI 0.65, 6.22) or DAS-28 (OR 1.70, 95\% CI 0.90, 3.17). No significant treatment effect was observed for tender and swollen joints, ESR, CRP, Health Assessment Questionnaire (HAQ) and pain. However, the MTX group did have a significant reduction in patient and assessor global scores and skin scores. The results of this study suggest that although MTX improved skin disease and symptoms, it did not improve inflammatory synovitis and thus did not have a true disease-modifying effect. This study was one of the first to enrol a significant proportion of patients with oligoarthritis with only 1 active joint required at entry. Some of the lack of response may relate to poorer sensitivity of established outcome measures in this group. Supplementary data from the study supports this with much higher responses seen in polyarticular patients $(n=142)$ compared to oligoarthritis $(n=79)$. There are a number of limitations to this trial, including a long recruitment time, mild disease at entry, low dose MTX with slow up-titration, high drop-out rate, and limited statistical power. There is also a lack of radiographic data to assess radiographic progression between MTX and 
placebo treated patients. Furthermore, although the trial examined peripheral arthritis and skin and nail disease, it failed to assess other domains within PsA such as enthesitis, dactylitis and axial involvement.

Kane et al. $2004{ }^{22}$ investigated the effect of MTX on synovial inflammation and gene expression in PsA, through synovial biopsies of an inflamed knee pre- and post-MTX treatment in 10 patients with active PsA. MTX was administered orally, with a median dose of $13.75 \mathrm{mg} /$ week. The median interval between synovial biopsies was 11.5 months. Post-MTX, there was a significant reduction in the Ritchie Articular Index, swollen joint count and Disease Activity Score, which corresponded to a significant decrease in CD3, CD4, CD8, CD68, E-selectin and ICAM immunohistochemical staining. Moreover, decreased proinflammatory cytokine expression was observed, however the effect was only significant for IL-8. Despite a significant reduction in synovial thickness, synovial hypervascularity was not significantly reduced and there remained a residual $\mathrm{T}$ cell infiltrate in all synovial biopsy tissues post-MTX. Taken together, these results suggest that MTX reduces, however does not abolish, synovial inflammation in PsA.

As MTX seems to have a beneficial effect in PsA in those who respond, Chandran et al. $2010^{23}$ investigated the genetic predictors of MTX efficacy in a longitudinal cohort of patients with PsA. Using response to MTX at 6 months as a cut off, they classified patients who achieved $a \geq 50 \%$ decrease in active inflamed joint count from baseline as responders. 119 patients were included in the effectiveness analysis, with a mean age of 44.1, mean duration of PsA of 9.3 years, and mean number of 15.5 actively inflamed joints. In this cohort, the authors tested 5 single nucleotide polymorphisms in genes involved in the folate pathway (MTHFR 677T, MTHFR 1298C, DHFR -473T, DHFR 35289A, RFC 80A) for association for MTX response. Using logistic regression and adjusting for concomitant medications, only the minor A allele of DHFR gene at +35289 was associated with increased odds of MTX response after 6 months of therapy (OR 2.99 CI 1.20, 7.55). MTX inhibits DHFR, which converts dihydrofolate to tetrahydrofolate. This study suggests there may be a genetic element to MTX response in PsA.

Should we use methotrexate to treat peripheral arthritis in PsA? There is a paucity of high-quality data from randomised controlled trials on the efficacy of MTX in treating peripheral arthritis in PsA. Available data are 
often derived from old studies, studies with small sample sizes or poor design, and studies limited by their observational nature with lack of appropriate controls. A recent Cochrane systematic review of 8 trials concluded that there is only low quality evidence for the efficacy of low-dose $(\leq 15 \mathrm{mg})$ oral MTX compared to placebo when taken for 6 months, in terms of PsARC, function, pain, and patient and physician global assessments of disease activity ${ }^{24}$. Studies thus far suggest that MTX use earlier in the disease process with higher doses appears to be associated with improved outcomes, and in a small proportion of patients, it is possible to achieve MDA. However, MTX therapy does not abolish radiographic progression or synovial inflammation, suggesting that it may not fully halt the underlying disease process.

When considering the efficacy of other csDMARDs in PsA, there is also a relative lack of data. Clegg et al. $1996{ }^{25}$ compared sulfasalazine (SSZ) to placebo in a pivotal randomised controlled trial of 221 patients with PsA. Although they showed that patients on sulfasalazine had significantly higher response rates compared to placebo, the overall response rate for sulfasalazine was not particularly high, at 57.8\%. In a subsequent Cochrane review meta-analysis ${ }^{26}$, the authors concluded that SSZ was effective in PsA but with a small effect size. Kaltwasser et al. $2004{ }^{27}$ published the results of the TOPAS trial, which compared leflunomide (LFN) to placebo in 190 patients with active PsA and psoriasis. At 24 weeks, the LFN group had significantly higher response rates as measured by the PsARC compared to placebo, with similar trends observed for ARC20 and PASI (Psoriasis Area and Severity Index) responses. However, LFN is not frequently used in clinical practice, with concerns over rare but serious adverse effects on the liver. Moreover, patients frequently do not tolerate the drug well.

There is limited data on the relative efficacy of different csDMARDs. In a single-centre retrospective analysis of 53 patients with PsA on MTX, SSZ and LFN ${ }^{28}$, all three agents led to consistent improvement in disease activity scores. However, SSZ seemed to exhibit greater efficacy compared to the other agents. The difficulties surrounding selecting the optimal csDMARD is reflected in the recent European League Against Rheumatism (EULAR) and Group for Research And Assessment of Psoriasis and Psoriatic Arthritis (GRAPPA) guidelines for the treatment of PsA ${ }^{29,30}$. Whilst both sets of guidelines advocate that in the majority of cases, a csDMARD should be the initial step in therapy, the recommendations are different. EULAR recommends MTX as the 
preferred option, with SSZ or LFN considered if there are contraindications to MTX. In contrast, GRAPPA advocates starting MTX, SSZ or LFN with no particular preference.

Moreover, there is clear evidence that biologics are superior to MTX in the treatment of PsA. In the RESPOND study ${ }^{10}$, a significantly higher proportion of patients receiving combination therapy with infliximab plus MTX achieved ACR20 by week 16 compared to patients on MTX monotherapy. In the SEAM-PsA study ${ }^{13}$, ACR20 and MDA response rates were significantly higher for patients on the etanercept monotherapy group compared to those on the MTX monotherapy group. These findings are echoed in a systematic literature review of randomised controlled trials by Kingsley et al. $2015^{31}$, which conclude that biologic agents appear to be more beneficial compared to csDMARDs. Indeed, the recently published American College of Rheumatology guidelines ${ }^{32}$ for treatment of PsA advocates starting a TNFi biologic as first-line, rather than csDMARDs, due to their increased efficacy.

\subsection{Dactylitis and Enthesitis}

There is very limited data on the efficacy of MTX to treat dactylitis and enthesitis in PsA as these domains have not been assessed well in older studies and there are no data from placebo-controlled studies. In an open-label observational study of 28 patients with active PsA and new-onset dactylitis, Healy and Helliwell $2007^{33}$ assessed the impact of changing disease modifying therapy on dactylitis. Although the majority of the patients were initiated on MTX (19/28), the results are not presented per treatment but for the cohort, which also includes patients on LFN, etanercept and hydroxychloroquine. At 3 and 6 months, the cohort exhibited an improvement in dactylitis across multiple measures used for dactylitis including the Leeds Dactylitis Index (LDI) basic. This corresponded to a PsARC and ACR20 response of 54\% and 50\% at 3 months, and of 51\% and $43 \%$ at 6 months, respectively. Given that the data for patients on MTX are not separately presented and the lack of controls, it is difficult to draw robust conclusions. However, the results do tentatively suggest that MTX may improve dactylitis in patients with PsA.

As part of the post-hoc analysis on the efficacy of MTX in PsA within the TICOPA study, Coates et al. $2016{ }^{11}$ also examined the effect of MTX on dactylitis and enthesitis. At 12 weeks, there was a significant reduction in 
the LDI basic score of $62.7 \%$ ( $\mathrm{p}=0.033$ ), with complete resolution of dactylitis seen in $37 / 59$ patients. Only 9 new cases of dactylitis were identified in patients without baseline involvement. In contrast, the median change in the enthesitis score was 0, independent of whether the Leeds Enthesitis Index, the IMPACT enthesitis score, or the Maastricht Ankylosing Spondylitis Enthesitis Score (MASES) was used. However, 38/148 patients had resolution of symptoms, with only 8 new cases of enthesitis identified which were not present at baseline. At 12 weeks, the proportions of patients with dactylitis and enthesitis were both significantly lower compared to baseline.

Similarly, in SEAM-PsA ${ }^{13}$, the authors observed an improvement in both dactylitis and enthesitis in the MTX monotherapy arm within the SEAM-PsA study. Using the LDI, 34.5\% of patients had an LDI $>0$ at baseline. At 24 weeks, the mean change (SE) from baseline was -128.8 (26.8), with $65.2 \%$ of patients having resolution of dactylitis. Using the Spondyloarthritis Research Consortium of Canada (SPARCC) enthesitis index, 67.3\% of patients had a score $>0$ at baseline. At 24 weeks, the mean change from baseline was -3.1, with $43 \%$ of patients with enthesitis experiencing resolution. Collectively, these results indicate that MTX may exert a beneficial effect on dactylitis and enthesitis in patients with PsA.

Should we use methotrexate to treat peripheral arthritis in PsA? Overall, there is very limited evidence for the efficacy of MTX and other csDMARDs in the treatment of dactylitis and enthesitis. In the SSZ study by Clegg et al. $1996{ }^{25}$, there was no significant difference in the dactylitis and enthesitis scores between patients treated with SSZ and placebo. In patients with predominantly entheseal disease, current guidelines ${ }^{29,30,32}$ recommend starting with biologic agents as the initial step rather than using MTX and other csDMARDs, as more robust evidence is available for biologics. However, this approach may need to be reconsidered given the recent results of the SEAM-PsA study ${ }^{13}$, which demonstrated no significant difference in the LDI and SPARCC enthesitis index between the etanercept monotherapy and MTX monotherapy groups at 24 weeks.

\subsection{Axial Disease}

MTX and other traditional csDMARDS are not effective in axial PsA, and thus MTX is not used to treat axial disease. Although there is a paucity of studies examining MTX in axial PsA, a recent Cochrane Systematic 
Review ${ }^{34}$ has been published summarising the evidence in ankylosing spondylitis (AS). The authors identified three randomised controlled trials in 116 participants with AS, and concluded that there was insufficient evidence to suggest that MTX therapy provided benefit in the treatment of AS.

Moreover, current measures of axial disease such as the Bath Ankylosing Spondylitis Disease Activity Index (BASDAI) does not discriminate well between peripheral and axial PsA. Taylor et al. $2004{ }^{35}$ studied two samples of patients with PsA, one from a disease register ( $n=133)$, and one from consecutive clinic attendees $(\mathrm{n}=47)$, and found that the BASDAI does not discriminate well between axial and peripheral PsA. FernandezSueiro et al. $2010{ }^{36}$ compared the BASDAI scores between 54 patients with peripheral PsA, 46 with axial PsA and 103 with primary AS. They found similar BASDAI scores in axial and peripheral PsA, corroborating the findings of Taylor et al. $2004^{35}$. The lack of a measure that discriminates between axial and peripheral disease within PsA poses challenges in clinical trials focusing on axial disease, as it is difficult to differentiate whether changes in outcomes are due to axial disease or other disease domains within PsA.

\subsection{Psoriasis}

MTX has consistently been shown to be effective in treating psoriasis, and is recommended as the first-line systemic csDMARD for moderate to severe psoriasis in European and US guidelines ${ }^{37,38}$. In the MIPA trial ${ }^{21}$, linear regression analysis showed that the MTX group had a significant reduction in PASI compared to placebo. In the post-hoc analysis of the TICOPA trial ${ }^{11}, 158$ patients had psoriasis and $27.2 \%$ of patients were able to achieve a PASI 75 response by week 12. In the MTX monotherapy group in SEAM-PsA ${ }^{13}, 66.1 \%$ of patients with $\geq 3 \%$ body surface area affected at baseline had an improvement by 24 weeks. Similarly, $65.7 \%$ of patients with $\geq 10 \%$ body surface area affected at baseline had an improvement by 24 weeks.

A recent meta-analysis of 11 studies by West et al. $2016{ }^{39}$ found that approximately $45 \%$ of patients with psoriasis on MTX achieve PASI 75 by 12 or 16 weeks compared to $4.4 \%$ of patients in the placebo group. However, there was substantial heterogeneity between the studies, with an $\mathrm{I}^{2}$ of $92.7 \%$. The authors conclude that although it appears that MTX is effective in treating psoriasis, it is difficult to quantify exactly how effective it is. As with studies examining the efficacy of MTX in peripheral arthritis, most studies on psoriasis 
are small with limited study designs and different dosing regimens. This highlights that compared to biologics, the evidence of MTX efficacy in psoriasis is limited.

As most studies of MTX efficacy in psoriasis utilise oral preparations, Warren et al. $2017{ }^{40}$ published the METOP study, which focuses on the efficacy of subcutaneous MTX in psoriasis. The rationale behind subcutaneous administration is based on prior observation that patients with poor response to oral MTX may benefit from subcutaneous MTX ${ }^{41}$. These findings corroborate with a study in rheumatoid arthritis ${ }^{42}$, which showed superiority of subcutaneous MTX over oral MTX after 24 weeks. The METOP study is a multicentre randomised double-blind placebo-controlled phase 3 trial investigating subcutaneous MTX in patients with moderate to severe plaque-type psoriasis. 120 MTX naïve patients with a diagnosis of chronic plaque psoriasis for $\geq 6$ months, and moderate to severe disease at baseline were randomised into receiving either MTX at a dose of $17.5 \mathrm{mg} /$ week $(\mathrm{n}=91)$ or placebo $(\mathrm{n}=29)$ for the first 16 weeks. At 8 weeks, the dose of MTX could be increased to $22.5 \mathrm{mg} /$ week if there had not been at least a 50\% reduction in baseline PASI. After 16 weeks, the study became open-label with all patients receiving MTX up to 52 weeks. At 16 weeks, a significantly higher proportion of patients in the MTX group achieved a PASI 75 response compared to placebo (41\% vs 10\%, RR 3.93, 95\% CI 1.31-11.91, p=0.0026). At 52 weeks, there was no significant difference between the proportions of patients achieving a PASI 75 response between the two groups, with all patients receiving MTX. Paired baseline and week 16 skin biopsies were available for 27 patients (placebo = 6, MTX PASI 75 non-responders = 7, MTX PASI 75 responders =14). Interestingly, MTX responders had prominent reductions in the numbers of skin-infiltrating CD3 positive T cells and CD11c positive dendritic cells, with week 16 numbers almost returning to values in normal skin. Moreover, MTX responders had reduced cutaneous mRNA levels of IL17A at week 16, whereas in MTX non-responders and placebo this was not significantly changed.

Collectively, these findings suggest that subcutaneous MTX is efficacious in treating psoriasis, and also shed light on the potential immunomodulatory mechanisms underlying the clinical response to MTX in psoriasis. However, the question of whether subcutaneous MTX is more efficacious compared to oral MTX in psoriasis remains unanswered.

\subsection{Nail disease}


Compared to psoriasis, there is very limited evidence for MTX in the treatment of psoriatic nail disease.

Moreover, the available evidence is limited by the short follow-up duration. The MIPA study ${ }^{21}$ did not find a treatment effect in either the MTX group or the placebo group at 3 and 6 months. In the TICOPA study ${ }^{11}, 117$ patients had nail involvement. At 12 weeks, the median change in mNAPSI score was -2 (interquartile range -8, 0 ), the median change in the nail plate score was 0 (interquartile range $-3.75,1$ ), and the median change in the nail bed score was -1 (interquartile range $-4.75,0$ ). In the study by Mease et al. ${ }^{13}, 65.1 \%$ of patients in the MTX monotherapy group had a mNAPSI $>0$ at baseline. At 24 weeks, the mean change from baseline was -1.1 , with $38.8 \%$ of patients achieving a score of 1 . The METOP study ${ }^{40}$ also examined the effect of MTX on nail psoriasis, using the NAPSI score of the worst fingernail as the target nail. At 16 weeks, the MTX group had a reduced NAPSI score, compared to no change in the placebo group. At 52 weeks, complete clearance of nail disease was seen in $14 \%$ of patients with active nail psoriasis at baseline. These results suggest that MTX may have a beneficial effect in nail disease. However, the response is likely to be slow, due to the slowing of nail growth.

\section{MTX and TNFi combination therapy}

MTX is frequently used in combination with TNFi however it is unclear whether this leads to better outcomes. Saad et al. $2010^{43}$ conducted an observational study of 595 patients with PsA from the British Society for Rheumatology Biologics Register (BSRBR). At 6 months, they found similar EULAR response rates in patients receiving TNFi monotherapy (79.5\%), TNFi in combination with MTX (78.1\%), and TNFi in combination with another DMARD (73.3\%). Fagerli et al. $2014{ }^{44}$ compared TNFi and MTX combination therapy (n=270) to TNFi monotherapy $(\mathrm{n}=170)$ in an observational study using the NOR-DMARD registry. In the combination therapy group, the mean MTX dose was $14.7 \mathrm{mg}$. At 3 and 6 months, the authors observed minimal differences between the two groups in the change in the physician and patient global scores. Looking only at patients with $\geq 1$ swollen joint at baseline, there was also no difference between the two groups in the change in DAS28 and DAPSA at 3 and 6 months. Interestingly, the combination therapy group had a trend towards improved drug survival at 3 years compared to the monotherapy group, with the results being most marked for infliximab. Moreover, the TNFi monotherapy group had an increased frequency of AEs, and also had a higher percentage discontinuing for loss or lack of efficacy compared to the combination therapy group. This therefore suggests 
that although concomitant MTX use with TNFi does not improve outcomes it may however improve TNFi survival.

MTX monotherapy, etanercept monotherapy, and MTX plus etanercept combination therapy were compared in the SEAM-PsA trial ${ }^{13}$, a phase 3 double blind randomised controlled trial of 851 patients with PsA naïve to both MTX and biologics. At week 24, ACR20 and MDA responses were similar between the etanercept monotherapy and combination therapy groups (ACR20: 60.9\% vs 65.0\%, MDA: 35.9\% vs 35.7\%). These findings corroborate results in previous studies, suggesting that concomitant MTX use does not improve outcomes compared to TNFi monotherapy. However, further studies with other TNFi biologics are required to investigate whether MTX improves TNFi survival, given that etanercept, as a fusion protein, may behave differently to other monoclonal antibodies in terms of long-term drug survival and risk of immunogenicity.

\section{Tolerability and adverse events}

When using MTX, safety as well as efficacy needs to be considered. Nikiphorou et al. $2014{ }^{45}$ conducted a single-centre retrospective study of the reasons for MTX discontinuation in patients with RA and PsA. They identified 762 patients with RA and 193 patients with PsA who received MTX, of which 260 and 71 discontinued MTX therapy, respectively. In both groups, the two most common reasons for discontinuation were adverse effects (RA: 77.5\%, PsA: 62.0\%), and inefficacy (RA: 12.4\%, PsA: 18.3\%). In patients with PsA who discontinued MTX due to adverse effects, the most common reasons were gastrointestinal symptoms (27.3\%), abnormal liver function tests (27.3\%), and respiratory symptoms (13.6\%). Only one patient discontinued due to neutropenia. At time of MTX discontinuation, the mean MTX in patients with PsA was 13.6 mg/week. In the MIPA trial ${ }^{21}$, common adverse events, defined as $>5 \%$ within a treatment arm, were nausea and vomiting, respiratory tract infections, and abnormal liver function tests. In the TICOPA study ${ }^{11}$, the most common adverse events reported were nausea, fatigue and liver abnormalities. Of 188 patients, only 14 discontinued MTX due to an adverse event (fatigue: 1, nausea: 5, gastrointestinal symptoms: 1, headache/migraine: 1, liver abnormalities: 4), although the MTX dose was modified or temporarily suspended in 77 patients. In a recent Cochrane systematic review of the use of MTX in PsA, the authors concluded that it remains uncertain whether MTX causes more harm than placebo, due to the small numbers of reported AEs ${ }^{24}$. 
Given that liver abnormalities are one of the major reasons for discontinuation of MTX, Montaudie et al. 201146 examined the incidence and risk factors for hepatotoxicity with MTX in patients with psoriasis. The authors concluded that the incidence of hepatic fibrosis reported in the literature is extremely variable.

\section{Conclusions}

MTX is widely used in the treatment of PsA, however, there is limited evidence for its efficacy in multiple domains (see Box 1). There is a paucity of comparative studies and strategy trials, which explains the differences between treatment recommendations for the initial step in PsA treatment. Some advocate MTX and other csDMARDs as the initial choice, whilst others advocate starting TNFi. There is no clear evidence for whether delaying access to biologics affects outcomes, and there is limited evidence on the optimal treatments in early disease. Given the heterogeneous nature of psoriatic disease, a personalised approach is required when deciding on the most appropriate therapy for individual patients.

It is clear that MTX is effective in skin psoriasis. It seems likely that MTX may improve joint symptoms in peripheral arthritis, with earlier use and higher initial doses being linked to better outcomes. MTX may also have a beneficial effect in dactylitis, enthesitis and psoriatic nail disease, but it is likely to exert a limited immunomodulatory effect rather than fully halting the disease process. Most studies on MTX in PsA are old and small scale with poor study designs, which limits the quality of the evidence. More recent studies have provided increasing evidence for the effectiveness of MTX in PsA, however these are not placebo-controlled.

Using MTX in combination with a TNFi does not seem to improve outcomes, however may increase TNFi survival. Moreover, MTX is generally well-tolerated, with the main adverse effects being gastrointestinal symptoms and liver abnormalities. However over time, a relatively small number of people remain on MTX monotherapy either due to lack of efficacy or intolerable side effects. Although MTX is less effective than the newer biologic therapies in treating PsA ${ }^{13}$, it is cheap and widely accessible, and therefore likely to retain an important role in the management of PsA, especially in more resource-limited healthcare settings. 


\section{References}

1. Bosch FV den, Coates L. Clinical management of psoriatic arthritis. The Lancet. 2018;391(10136):2285-2294

2. Coates LC, Moverley AR, McParland L, et al. Effect of tight control of inflammation in early psoriatic arthritis (TICOPA): a UK multicentre, open-label, randomised controlled trial. The Lancet. 2015;386(10012):2489-2498.

3. Coates LC, Fransen J, Helliwell PS. Defining minimal disease activity in psoriatic arthritis: a proposed objective target for treatment. Annals of the Rheumatic Diseases. 2010;69(01):48-53.

4. Ceponis A, Kavanaugh A. Use of methotrexate in patients with psoriatic arthritis. Clin Exp Rheumatol. 2010 Sep-Oct;28(5 Suppl 61):S132-7.

5. Elmamoun M, Chandran V. Role of Methotrexate in the Management of Psoriatic Arthritis. Drugs. 2018;78(6):611-619

6. Black RL, O’Brien WM, Scott EJV, Auerbach R, Eisen AZ, Bunim JJ. Methotrexate Therapy in Psoriatic Arthritis: Double-Blind Study on 21 Patients. JAMA. 1964;189(10):743-747.

7. Chandran V, Schentag CT, Gladman DD. Reappraisal of the effectiveness of methotrexate in psoriatic arthritis: results from a longitudinal observational cohort. The Journal of Rheumatology. 2008;35(3):469-471.

8. Scarpa R, Peluso R, Atteno M, et al. The effectiveness of a traditional therapeutical approach in early psoriatic arthritis: results of a pilot randomised 6-month trial with methotrexate. Clin Rheumatol. 2008;27(7):823-826.

9. Lie E, Heijde D van der, Uhlig T, et al. Effectiveness and retention rates of methotrexate in psoriatic arthritis in comparison with methotrexate-treated patients with rheumatoid arthritis. Annals of the Rheumatic Diseases. 2010;69(4):671-676.

10. Baranauskaite A, Raffayová H, Kungurov NV, et al. Infliximab plus methotrexate is superior to methotrexate alone in the treatment of psoriatic arthritis in methotrexate-naive patients: the RESPOND study. Annals of the Rheumatic Diseases. 2012;71(4):541-548. 
11. Coates LC, Helliwell PS. Methotrexate Efficacy in the Tight Control in Psoriatic Arthritis Study. The Journal of Rheumatology. 2016;43(2):356-361.

12. Willkens RF, Williams HJ, Ward JR, et al. Randomized, Double-Blind, Placebo Controlled Trial of Low-Dose Pulse Methotrexate in Psoriatic Arthritis. Arthritis \& Rheumatism. 1984;27(4):376-381.

13. Mease PJ, Gladman DD, Collier DH, Ritchlin CT, Helliwell PS, Liu L, Kricorian GJ, Chung JB. Etanercept and Methotrexate As Monotherapy or in Combination in Patients with Psoriatic Arthritis: A Phase 3, Double-Blind, Randomized Controlled Study [abstract]. Arthritis Rheumatol. 2018; 70 (suppl 10)

14. Coates LC, Helliwell PS. Validation of minimal disease activity criteria for psoriatic arthritis using interventional trial data. Arthritis Care Res (Hoboken). 2010;62:965-9

15. Coates LC, Cook R, Lee KA, Chandran V, Gladman DD. Frequency, predictors, and prognosis of sustained minimal disease activity in an observational psoriatic arthritis cohort. Arthritis Care Res (Hoboken). 2010;62:970-6.

16. Kavanaugh A, van der Heijde D, Beutler A, Gladman D, Mease P, Krueger GG, et al. Radiographic progression of patients with psoriatic arthritis who achieve minimal disease activity in response to golimumab therapy: results through 5 years of a randomized, placebo-controlled study. Arthritis Care Res (Hoboken). 2016;68:267-74.

17. Queiro R, Canete JD, Montilla C, Abad M, Montoro M, Gomez S, et al. Minimal disease activity and impact of disease in psoriatic arthritis: a Spanish cross-sectional multicenter study. Arthritis Res Ther. 2017;19:72.

18. Cantini F, Niccoli L, Nannini C, et al. Frequency and duration of clinical remission in patients with peripheral psoriatic arthritis requiring second-line drugs. Rheumatology (Oxford). 2008;47(6):872-876.

19. Abu-Shakra M, Gladman DD, Thorne JC, Long J, Gough J, Farewell VT. Longterm methotrexate therapy in psoriatic arthritis: clinical and radiological outcome. J Rheumatol. 1995;22(2):241-245.

20. Eder L, Thavaneswaran A, Chandran V, Gladman DD. Tumour necrosis factor $\alpha$ blockers are more effective than methotrexate in the inhibition of radiographic joint damage progression among patients with psoriatic arthritis. Ann Rheum Dis. 2014;73(6):1007-1011.

21. Kingsley GH, Kowalczyk A, Taylor H, et al. A randomized placebo-controlled trial of methotrexate in psoriatic arthritis. Rheumatology (Oxford). 2012;51(8):1368-1377. 
22. Kane D, Gogarty M, O’leary J, et al. Reduction of synovial sublining layer inflammation and proinflammatory cytokine expression in psoriatic arthritis treated with methotrexate. Arthritis Rheum. 2004;50(10):3286-3295.

23. Chandran V, Siannis F, Rahman P, Pellett FJ, Farewell VT, Gladman DD. Folate Pathway Enzyme Gene Polymorphisms and the Efficacy and Toxicity of Methotrexate in Psoriatic Arthritis. The Journal of Rheumatology. 2010;37(7):1508-1512.

24. Wilsdon TD, Whittle SL, Thynne TR, Mangoni AA. Methotrexate for psoriatic arthritis. Cochrane Database Syst Rev. 2019 Jan 18;1:CD012722.

25. Clegg DO, Reda DJ, Weisman MH, et al. Comparison of sulfasalazine and placebo in the treatment of ankylosing spondylitis. A department of veterans affairs cooperative study. Arthritis \& Rheumatism. 1996;39(12):2004-12.

26. Jones G, Crotty M, Brooks P. Interventions for psoriatic arthritis. Cochrane Database Syst Rev. 2000;(3):CD000212.

27. Kaltwasser JP, Nash P, Gladman D, et al. Efficacy and safety of leflunomide in the treatment of psoriatic arthritis and psoriasis: A multinational, double-blind, randomized, placebo-controlled clinical trial. Arthritis \& Rheumatism. 2004 Jun;50(6):1939-50.

28. Roussou E, Bouraoui A. Real-life experience of using conventional disease-modifying anti-rheumatic drugs (DMARDs) in psoriatic arthritis (PsA). Retrospective analysis of the efficacy of methotrexate, sulfasalazine, and leflunomide in PsA in comparison to spondyloarthritides other than PsA and literature review of the use of conventional DMARDs in PsA. Eur J Rheumatol. 2017 Mar;4(1):1-10.

29. Gossec L, Smolen JS, Ramiro S, et al. European League Against Rheumatism (EULAR) recommendations for the management of psoriatic arthritis with pharmacological therapies: 2015 update. Ann Rheum Dis. 2016 Mar;75(3):499-510.

30. Coates LC, Kavanaugh A, Mease PJ, et al. Group for Research and Assessment of Psoriasis and Psoriatic Arthritis 2015 Treatment Recommendations for Psoriatic Arthritis. Arthritis \& Rheumatology (Hoboken, NJ). 2016;68(5):1060-71.

31. Kingsley GH, Scott DL. Assessing the effectiveness of synthetic and biologic disease-modifying antirheumatic drugs in psoriatic arthritis - a systematic review. Psoriasis (Auckl). 2015;5:71-81. 
32. Singh JA, Guyatt G, Ogdie A, et al. Special Article: 2018 American College of Rheumatology/National Psoriasis Foundation Guideline for the Treatment of Psoriatic Arthritis. Arthritis Care Res (Hoboken). 2019 Jan;71(1):2-29.

33. Healy PJ, Helliwell PS. Measuring dactylitis in clinical trials: which is the best instrument to use? The Journal of Rheumatology. 2007;34(6):1302-1306.

34. Chen J, Veras MMS, Liu C, Lin J. Methotrexate for ankylosing spondylitis. Cochrane Database Syst Rev. 2013;(2):CD004524.

35. Taylor WJ, Harrison AA. Could the Bath Ankylosing Spondylitis Disease Activity Index (BASDAI) be a valid measure of disease activity in patients with psoriatic arthritis? Arthritis Rheum. 2004;51(3):311315.

36. Fernández-Sueiro JL, Willisch A, Pértega-Díaz S, et al. Validity of the bath ankylosing spondylitis disease activity index for the evaluation of disease activity in axial psoriatic arthritis. Arthritis Care Res (Hoboken). 2010;62(1):78-85.

37. Psoriasis: Recommendations for methotrexate | American Academy of Dermatology. https://www.aad.org/practicecenter/quality/clinical-guidelines/psoriasis/systemicagents/recommendations-for-methotrexate. Accessed January 13, 2019.

38. Warren RB, Weatherhead SC, Smith CH, et al. British Association of Dermatologists' guidelines for the safe and effective prescribing of methotrexate for skin disease 2016. Br J Dermatol. 2016;175(1):23-44.

39. West J, Ogston S, Foerster J. Safety and Efficacy of Methotrexate in Psoriasis: A Meta-Analysis of Published Trials. PLoS ONE. 2016;11(5):e0153740.

40. Warren RB, Mrowietz U, von Kiedrowski R, et al. An intensified dosing schedule of subcutaneous methotrexate in patients with moderate to severe plaque-type psoriasis (METOP): a 52 week, multicentre, randomised, double-blind, placebo-controlled, phase 3 trial. Lancet. 2017;389(10068):528537.

41. Yesudian PD, Leman J, Balasubramaniam P, et al. Effectiveness of Subcutaneous Methotrexate in Chronic Plaque Psoriasis. J Drugs Dermatol. 2016 Mar;15(3):345-9.

42. Braun J, Kästner P, Flaxenberg P, et al. Comparison of the clinical efficacy and safety of subcutaneous versus oral administration of methotrexate in patients with active rheumatoid arthritis: results of a six- 
month, multicenter, randomized, double-blind, controlled, phase IV trial. Arthritis Rheum 2008; 58: 73-81.

43. Saad AA, Ashcroft DM, Watson KD, et al. Efficacy and safety of anti-TNF therapies in psoriatic arthritis: an observational study from the British Society for Rheumatology Biologics Register. Rheumatology (Oxford). 2010;49(4):697-705.

44. Fagerli KM, Lie E, van der Heijde D, et al. The role of methotrexate co-medication in TNF-inhibitor treatment in patients with psoriatic arthritis: results from 440 patients included in the NOR-DMARD study. Ann Rheum Dis. 2014;73(1):132-137.

45. Nikiphorou E, Negoescu A, Fitzpatrick JD, et al. Indispensable or intolerable? Methotrexate in patients with rheumatoid and psoriatic arthritis: a retrospective review of discontinuation rates from a large UK cohort. Clin Rheumatol. 2014;33(5):609-614.

46. Montaudié H, Sbidian E, Paul C, et al. Methotrexate in psoriasis: a systematic review of treatment modalities, incidence, risk factors and monitoring of liver toxicity. J Eur Acad Dermatol Venereol. 2011;25 Suppl 2:12-18. 